\title{
The Feasibility of Integrating Flexible Sensor and Virtual Self-Organizing DC Grid Model in an Embedded System for Blood Leakage Detection During Dialysis Treatment
}

\author{
${ }^{1}$ Ping-Tzan Huang, ${ }^{5}$ Chih-Cheng Kao, ${ }^{2}$ Chien-Ming Li, ${ }^{3}$ Yueh-Ren Ho, ${ }^{4}$ Wei-Ling Chen, ${ }^{4}$ Ming-Jui Wu, \\ and ${ }^{5}$ Chia-Hung Lin \\ ${ }^{1}$ Department of Electrical Engineering, National Tsing Hua University, Hsinchu, 30013, Taiwan. \\ E-mail: huangpt317@gmail.com. \\ ${ }^{2}$ Division of Infectious Diseases, Department of Medicine of Chi Mei Medical Center, Tainan City, 710, Taiwan. \\ E-mail: 235813cmli@gmail.com. \\ ${ }^{3}$ Department of Biochemistry, Medical College of National Cheng Kung University Tainan City, 70101, Taiwan. \\ E-mail: yrho@mail.ncku.edu.tw. \\ ${ }^{4}$ Department of Engineering and Maintenance, Kaohsiung Veterans General Hospital, Kaohsiung City, 81362, Taiwan. \\ E-mail: lynnchen.k@gmail.com. \\ ${ }^{4}$ Department of Internal Medicine, Kaohsiung Veterans General Hospital, Tainan Branch, Tainan City, 71051, Taiwan. \\ E-mail: wmr216@yahoo.com.tw. \\ ${ }^{5}$ Department of Electrical Engineering, Kao-Yuan University, Kaohsiung City, 82151, Taiwan. \\ E-mail: eechl53@gmail.com,t20005@cc.kyu.edu.tw,
}

\begin{abstract}
Blood leakage or serious blood loss is a frequent life-threatening complication that occurs during dialysis treatment. When these events occur, it takes only a few minutes for blood loss in an adult patient, resulting in mortality. As an early-warning design, this study proposes integrating a flexible sensor and a virtual self-organizing DC (Direct Current) grid model for blood leakage detection. A flexible sensor is made using a screen printing technique, and a self-organizing algorithm is used to automatically construct a virtual DC grid from an existing grid to an extending grid. That is, a virtual blood leakage detector can be modeled, which is intended for detection of blood leakage or serious blood loss. The feasibility is verified, and the model is implemented in an embedded system.
\end{abstract}

Index Terms-Blood Leakage, Flexible Sensor, Self Organizing, DC (Direct Current), Virtual DC Grid Model.

\section{Introduction}

According to the 2016 statistics, in Taiwan, more than 80,000 patients with end-stage renal disease and chronic kidney failure have received dialysis therapy. In a routine dialysis treatment, certain complications occur frequently, including vascular access stenosis [1], venous needle dislodgment, body fluid/blood leakage, and serious blood loss [2-3]. Among these events, blood leakage or serious blood loss is a life-threatening complication. With blood flow rates of $400-500 \mathrm{ml} / \mathrm{min}$, it takes only a few minutes to lose more than $40 \%$ of the adult blood volume. In a lecture in 2012 [4], the American Nephrology Nurses' Association (ANNA) venous needle dislodgement (VND) survey reports revealed that more than $75 \%$ of the surveyed candidates indicated that they had observed a VND event in a hemodialysis room with more than $8 \%$ having observed five events or more in the last 5 years. The reports also revealed that more than half of the surveyed candidates concerned about VND or serious blood loss indicated that they were concerned about VND very often $(>30 \%)$ or often $(>20 \%)$. Six risk levels were identified in the ANNA

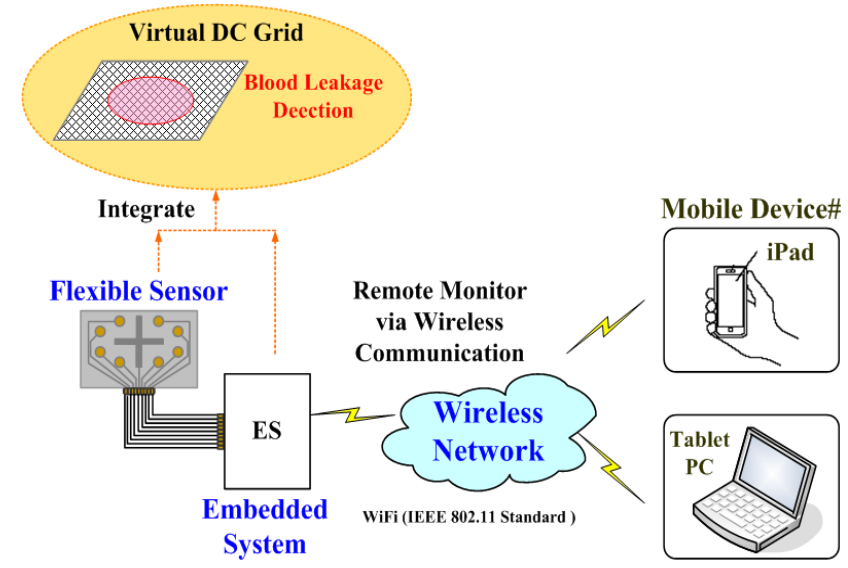

Figure 1. The proposed configuration of an integrating flexible sensor and virtual DC grid

VND survey results [3], and practice recommendations were also implemented to prevent VND [3, 5-7], including selecting an adequate access cannulation site, which serves a dual purpose, taping the needles securely, assuring access visibility, using a safety dialysis machine, and monitoring the devices.

In addition, various alarms have been built into a hemodialysis machine that can warn system malfunction through pressure (pump pull and push), blood leaks, pump flow, and temperature monitors. Measuring venous pressure helps in detecting VND, whereas pressure drops act to stop the roller pump. Pad sensors, optical sensors, and wetness sensors [8-10] can also be used as an early warning detector, such as the customized product Redsense ${ }^{\circledR}$ monitor (CE mark, Halmstad, Sweden, approved by the FDA) for high-risk patients and home HD. It is placed at the access cannulation sites around the venous and arterial needles to detect blood leakage or blood loss. It relies on an optical sensor (infrared light) and is not sensitive to clear but conductive fluids such as saline. A pad sensor [9] comprises upper / lower membrane layers and an electrical circuit connection. When blood comes in contact with the circuit connection, electrical connection changes produce electrical 

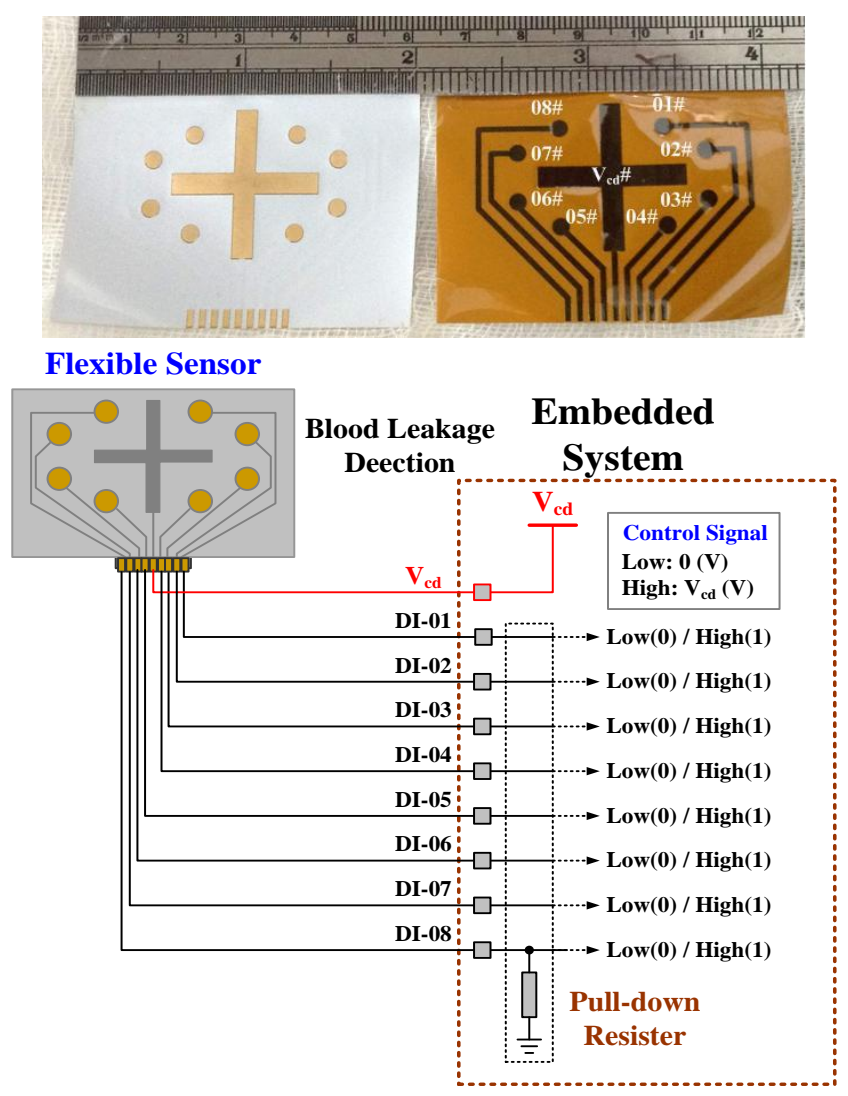

Figure 2. The configuration of proposed flexible sensor

signals that are sent to the analytical circuit (alarm unit) or analytical method [10]. These base membrane layers need to be made of a medical-grade and biocompatible material. Electromagnetic compatibility (EMC), circuit electrical performance, and electrical tests should be validated (IEC 60601-1, medical design standards) for medical electrical equipments and compliances. Wetness sensors are sensitive to not only blood but also saline and other fluids in the patient's body environment [2]. However, these alarm tools cannot stop the blood pump as there is no interface with the hemodialysis machine.

Hence, this study proposed integrating a flexible sensor [11-13] and a virtual DC grid model [14-15] for body fluid / blood leakage detection during dialysis treatment, as shown in Figure 1. The virtual DC grid model with a sensing unit and a wireless communication [16] can be implemented in an embedded system. The virtual DC grid model is a manner to design a virtual self-organizing analytical circuit/ alarm unit. It can automatically build up an impedance matrix by adding the analytical circuit's connecting branches one at a time to construct a complete DC grid that is formed from an existing impedance matrix to extend a new large-scale impedance matrix. Its advantage is that it can be easily implemented on a digital tablet (PC) or an intelligent mobile devices. The so-called "rake equivalent circuit" is applied for short circuit analysis in a grid using the current injection method [17-18]. A flexible sensor consists of an electrical connection circuit and is made using a screen printing technique with elastic and metallic materials. A detector integrating a flexible sensor and a virtual DC grid can then be implemented into an embedded

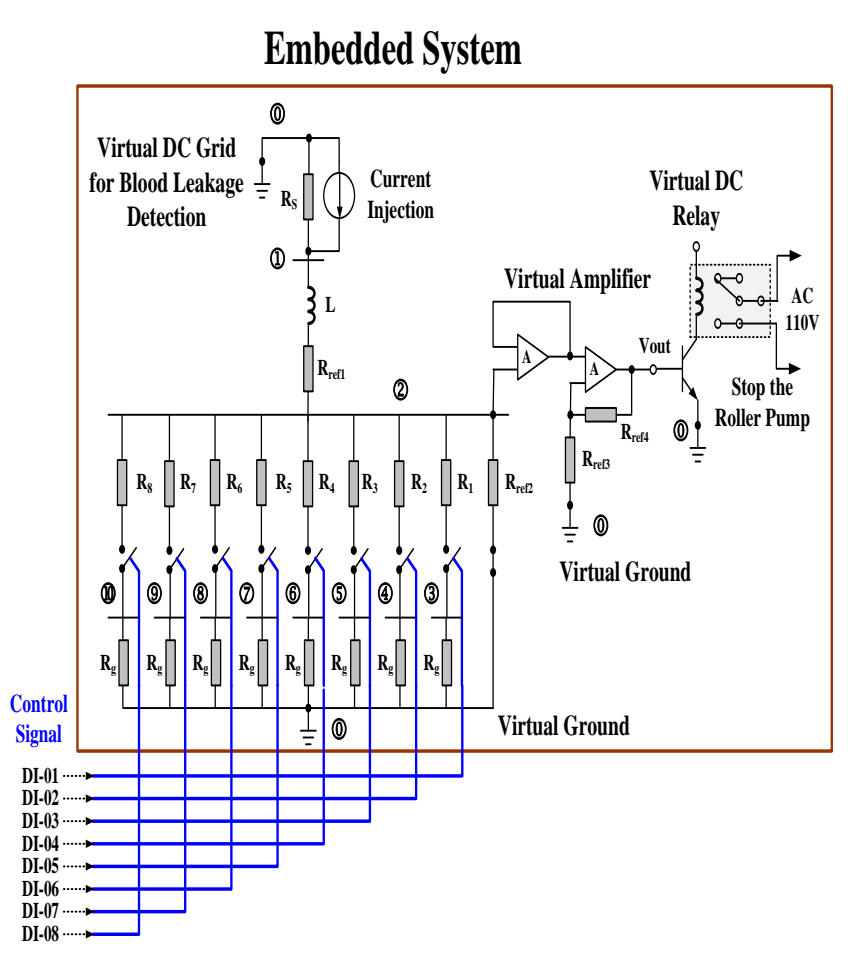

Figure 3. The configuration of proposed virtual DC grid for blood leakage detection

system and WiFi wireless local area network (WLAN, IEEE 802.11 Standards) [16]. The feasibility of the proposed model is verified in this study.

\section{Methodology}

\section{A. The Proposed Configuration of Flexible Sensor}

Flexible sensors have been applied in several fields, including biomedical engineering, industrial automation controls, robotic sensing controls, communication security, human-robot interaction, and human-machine interfacing [11-13]. Capacitive-based, piezoresistive-based, and piezoelectric-based sensors are commonly used to measure pressure, force, and temperature and for tactile examination. They are thin, soft, flexible, and stretchable and can integrate a mobile and a wearable device on the human body. For healthcare applications, noninvasive sensing systems and health-monitoring appliances are widely used for clinical needs and biomedical diagnostic procedures. An advantage of the flexible sensor is that it can be placed anywhere on the patient's body.

Therefore, a printed circuit board (PCB) was mounted in a flexible planar, with a size of $60 \mathrm{~mm} \times 40 \mathrm{~mm}$ body-fluid -sensitive element with 9 nodes. It is a configuration of an 8-position switch that manipulates the open and closed states through blood leakage / serious blood loss. Interface conductors connect directly to embedded system connectors (digital inputs, DIs) without using any additional components, because of using the pull-up on MXP (port) connectors or pull-down on MSP (port) connectors. MSP connectors (DI-01 DI-08) are used to measure $0-3.3 \mathrm{~V}$ signal [16]. Each connector has an internal $40 \mathrm{k} \Omega$ pull-down 
resister. For a constant voltage source, $\mathrm{V}_{\mathrm{cd}}=+3.3 \mathrm{~V}$, eight connecting conductors will appear as short circuit in any position. On the MSP connectors, each DI has a connection from $\mathrm{V}_{\mathrm{cd}}$ to pull-down resister, as shown in Figure 2. Hence, each DI has one terminal tied high to the voltage, $\mathrm{V}_{\mathrm{cd}}$, and then the numerical values or binary patterns can be obtained. The eight-bit binary patterns can be defined as (1)

$$
C_{l}=\left\{\begin{array}{l}
1, \text { if } V_{l}=V_{c d}(\text { High }) \\
0, \text { if } V_{l}=0(L \circ \mathrm{w}
\end{array}, l=1,2,3, \ldots, 8\right.
$$

where the 8-bit binary patterns are control signals, which are used to control the resister, $R_{g}$, tied to the virtual ground, as shown in Figure 3.

Then, a WiFi WLAN is used for linking mobile devices (smart phones, personal digital assistant, or iPad) and portable computers (laptops), while operating on $2.4-\mathrm{GHz}$ industrial, science, and medical frequency bands. It is employed to transit the control signals for portable devices with short-range communications in common household and mobile appliances.

\section{B. Virtual DC Grid Model in an Embedded System}

As shown in Figure 3, consider a virtual DC grid consisting of 10 nodes $(n=10)$ and 11 branches $(b=11)$, whose DC grid has the following connecting matrix, $M_{C}$, as follows:

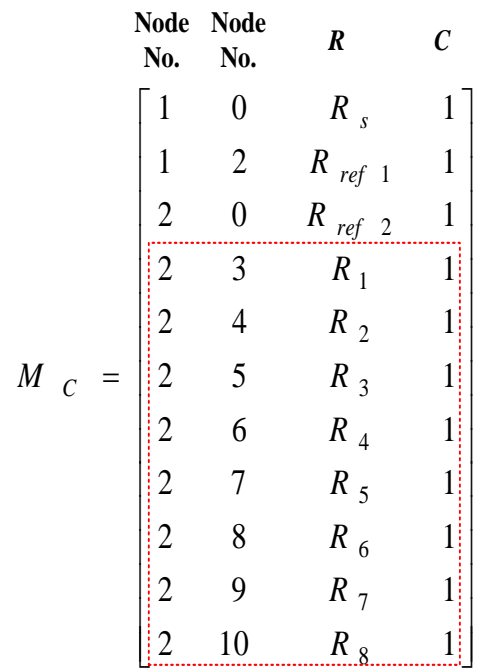

where Column 1\# and Column 2\# are the branches with resisters, including additional resisters between two nodes (1)\# - (2)\# to (3)\# - (10\#), and an additional resister from one node to the reference node ((0)\#); Column 3\# indicates the resisters in the DC grid; and Column 4\# describes the connecting state, the symbol " $C=1$ (High)" indicates the connecting state; otherwise, it is symbol " $C=0$ (Low)."

The constant $R_{s}$ is the internal resister in the current source, $I$. Resisters, $R_{r e f 1}$ and $R_{f e f 2}\left(R_{r e f 1}=2 \times R_{f e f 2}\right)$, are used to determine the normal output voltage, $V_{\text {out }}$, using the voltage divider rule, as follows:

$$
V_{\text {out }}=\left(1+\frac{R_{r_{\text {ef }}}}{R_{r_{\text {e } 3}}}\right) \times\left(\frac{R_{r_{\text {e⿻ }}}}{R_{r_{\text {elf }}}+R_{r_{\text {ef }}}}\right) \times\left(I \times R_{s}\right)
$$

where $\left(1+R_{\text {ref } 4} / R_{\text {ref } 3}\right)$ is the magnification of a virtual amplifier. The voltage level is $3.3 \mathrm{~V}$ for further wireless communication application via the WiFi WLAN (IEEE
802.11 Standard). Constants $R_{1}$ to $R_{8}$ are the branch resisters. The ground connection matrix, $M_{g}$, is defined as follows:

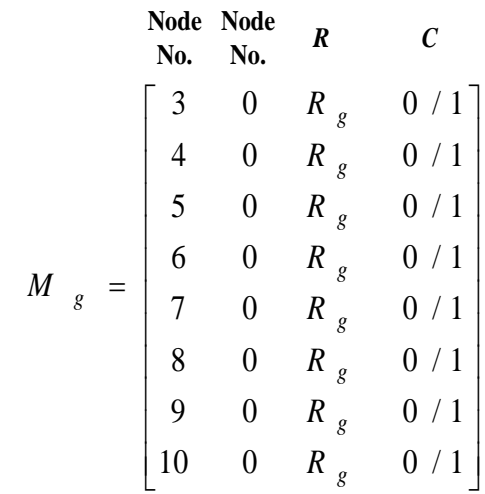

where Column 1\# and Column 2\# describe the short circuit between one node to virtual ground (grounded branch: $g=$ 8 ), due to blood leakage. Constant $R_{g}$ is the virtual ground resistor, as described below:

$$
R_{g}=R_{l}=R_{\text {ref }}, \quad l=1,2,3, \ldots, 8
$$

When the blood leakage is detected by the flexible sensor, the control signal will be " $C=1$ " and the grounded branch will be connected. The connecting matrix and the ground connection matrix are combined, whose virtual DC grid has the following admittance matrix, as shown below [14, 17]:

$$
\begin{aligned}
& M=\left[\begin{array}{l}
M_{C} \\
-- \\
M_{g}
\end{array}\right]_{h \times 4}, \quad h=1,2,3, \ldots,(b+g) \\
& Y=\left[Y_{i j}\right] \in R^{n \times n}, \quad Y_{i j}=\left\{\begin{array}{l}
\left(y_{i j}+\sum_{k \neq i}^{n} y_{i k}\right), \quad \text { if } i=j \\
-y_{i j}, \quad \text { if } i \neq j
\end{array}\right. \\
& y_{i j}=\frac{1}{M_{h 3}}, \quad i=1,2,3, \ldots, n, \quad j=1,2,3, \ldots, n,
\end{aligned}
$$

where node number, $n=10$; branch number, $b=11$; and grounded branch, $g=8 ; y_{i j}$ is the admittance $\left(y_{i i} \neq 0\right)$ between the node, $i$, and node, $j$.

In the virtual DC grid, only the current source is injected into node (1)\#, and the nodal equations for the grid are:

$$
\left.Y V=I \Rightarrow[Y]_{10 \times 10}\left[\begin{array}{c}
V_{1} \\
V_{2} \\
V_{3} \\
\vdots \\
V_{10}
\end{array}\right]_{10 \times 1} \begin{array}{c}
I_{S} \\
0 \\
0 \\
0 \\
0 \\
0
\end{array}\right] 10 \times 1
$$

$$
Z I=V, Z=Y^{-1}
$$

where $I_{S}$ is the injection current into node (1)\#; $Z$ is the impedance matrix; and both $Z$ and $Y$ are symmetric matrices; the nodal voltages at each node can be obtained using the current injection method [18-19], as follows:

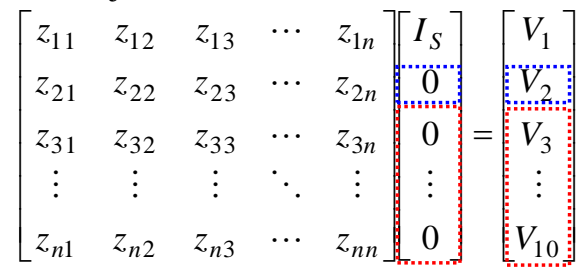

where nodal voltages, $V_{3}$ to $V_{10}$, are used to identify the 


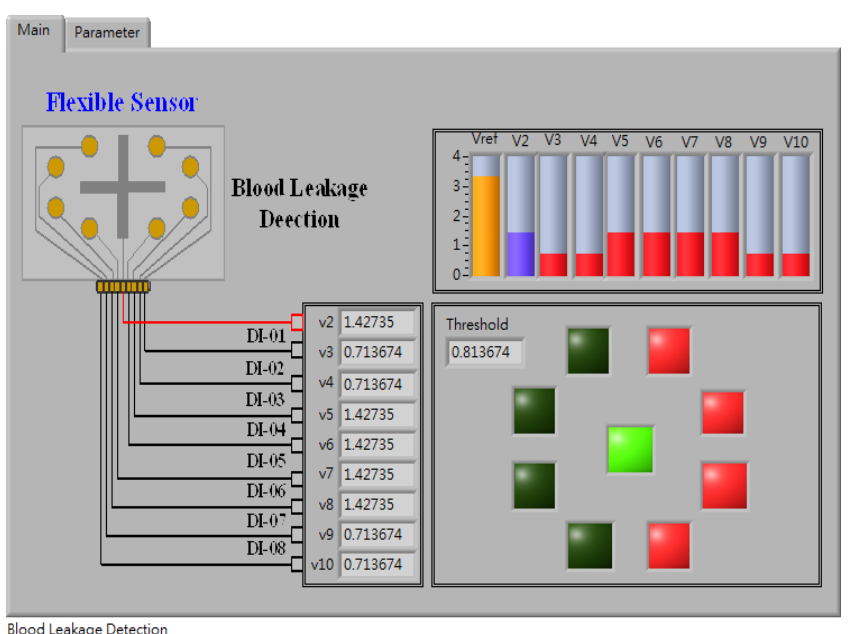

Figure 4. The graphical programming user interface

blood leakage, while their grasp drops have blood leakage occurrence, as follows:

$$
\begin{aligned}
V_{\min } & =\min \left\{V_{3}, V_{4},, \ldots, V_{10}\right\}, V_{\max }=\max \left\{V_{3}, V_{4},, \ldots, V_{10}\right\} \\
V_{\text {ref }} & =\frac{1}{2}\left(V_{\min }+V_{\max }\right) \\
S_{j} & = \begin{cases}0, & V_{j}>V_{\text {ref }} \\
1, & V_{j} \leq V_{\text {ref }}\end{cases}
\end{aligned}
$$

where state variable, $S_{j}=1$, indicates that one or more nodes have detected a blood leakage event. Therefore, signal " 1 " acts to drive an alarm unit. In addition, the power flow equations of the grounded branches can be represented as

$$
P=V_{i} \times \sum_{l=1}^{8}\left(\frac{V_{i}}{R_{l}}\right), \quad i=3,4,5, \ldots, 10, \quad R_{l} \neq 0
$$

where grounded branch, $R_{l} \neq 0$, while the connecting state " $C=1$ "; otherwise " $C=0$ " indicates that the grounded branch is not connected, and resister, $R_{l}=0$. When several grounded branches are connected, the power flow will increase.

There are two grasp changes that can be used to quantify the level of blood leakage and can be summarized as follows:

- nodal voltage drops: nodal voltages, $V_{3}$ to $V_{10}$, are drops, while one or more sensing points in a flexible sensor have detected blood leakage using equations (12) and (13),

- power flow increases: one or more ground branches are connected, resulting in power flow increases.

\section{Experimental Results}

The proposed prototype detection model has been developed in an embedded system (National Instruments ${ }^{\mathrm{TM}}$ myRIO-1900, Austin, Texas, U.S.A). The virtual DC grid model was coded as a "self-organizing algorithm" in an embedded system, including matrix methods for large-scale network and a current injection method. The flexible sensor $(60 \mathrm{~mm} \times 40 \mathrm{~mm})$ was made using a screen printing technique with elastic and metallic materials. As shown by the connecting topology in Figure 3, the DC grid has 10 (a)

$\begin{array}{lll}\text { (1) Normal Condition } & \text { (2) } 1 \text { Node Detection } & \text { (3) } 2 \text { Nodes Detection }\end{array}$

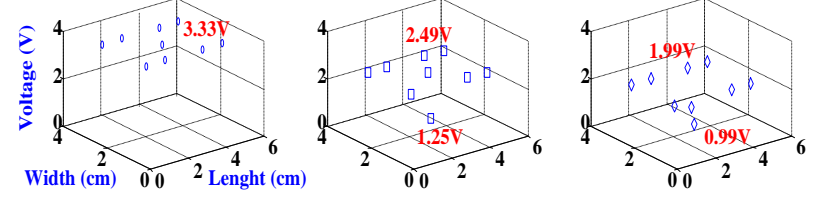

(4) 3 Nodes Detection

(5) 4 Nodes Detection

(6) 5 Nodes Detection
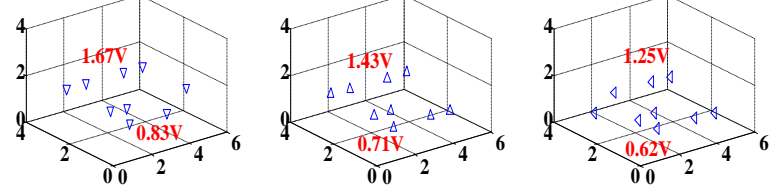

(7) 6 Nodes Detection

(8) 7 Nodes Detection

(9) 8 Nodes Detection
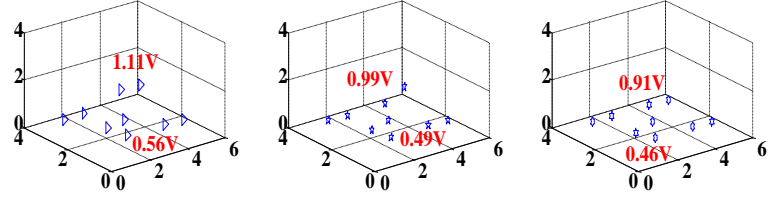

(b)

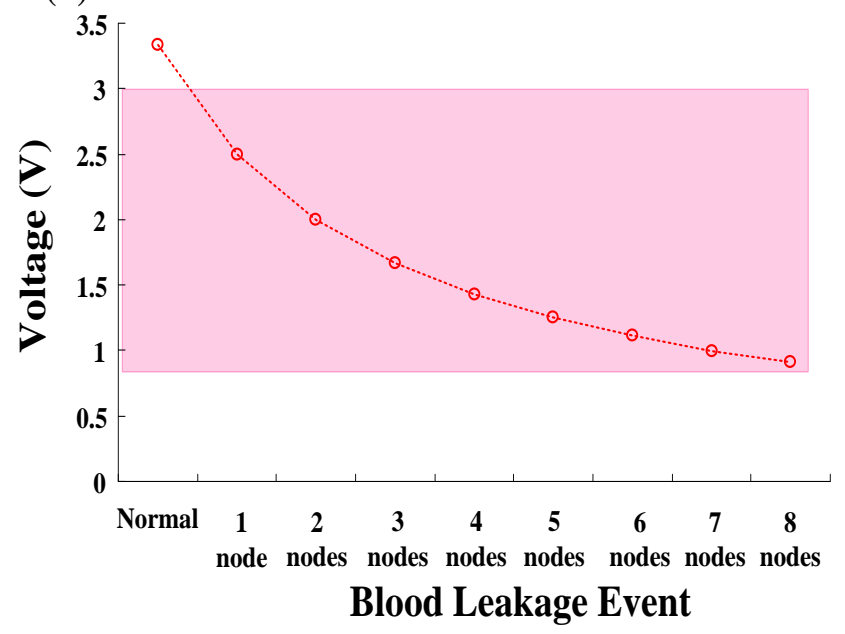

Figure 5. Experimental results. (a) Nodal voltage distributions in a $60 \mathrm{~mm} \times 40 \mathrm{~mm}$ region, (b) Nodal voltages, $V_{2}$, versus blood leakage events at node $2 \#$

nodes and its connecting injection current, inductance, and resisters are shown as follows:

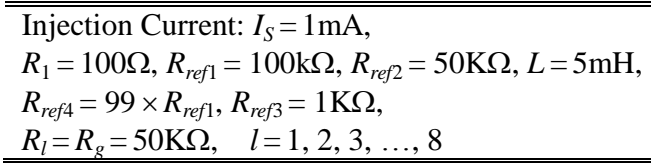

Hence, considering the connecting states, $C$, of the connecting matrices, $M_{C}$ and $M_{g}$, the original impedance matrix, $Z$, was constructed at the top of the next page, where the square matrix, $Z$, was a symmetric and nonsingular matrix that was equal to transpose, $Z=Z^{T}$ and $|Z| \neq 0$. The connecting states were obtained from the flexible sensor. The proposed prototype model was designed using a LabVIEW graphical programming user interface $\left(\mathrm{NI}^{\mathrm{TM}}\right.$ Corporation, Austin, Texas, U.S.), as shown in Figure 4, and was carried out in a development platform, such as a tablet $\mathrm{PC}$ or mobile intelligent vehicles. 


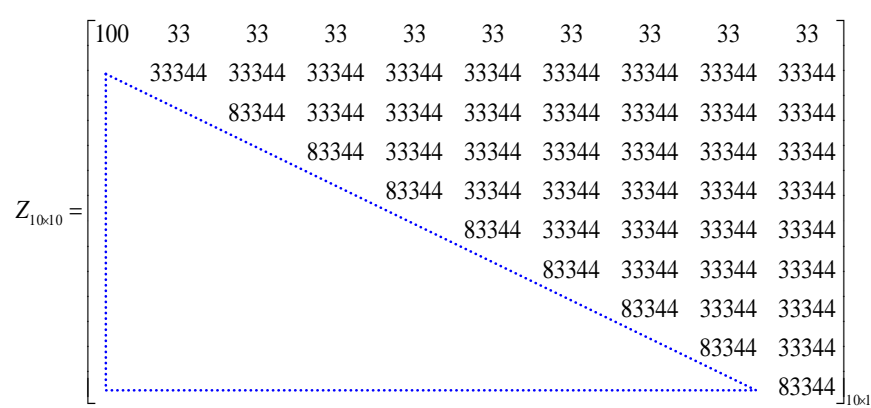

(a)

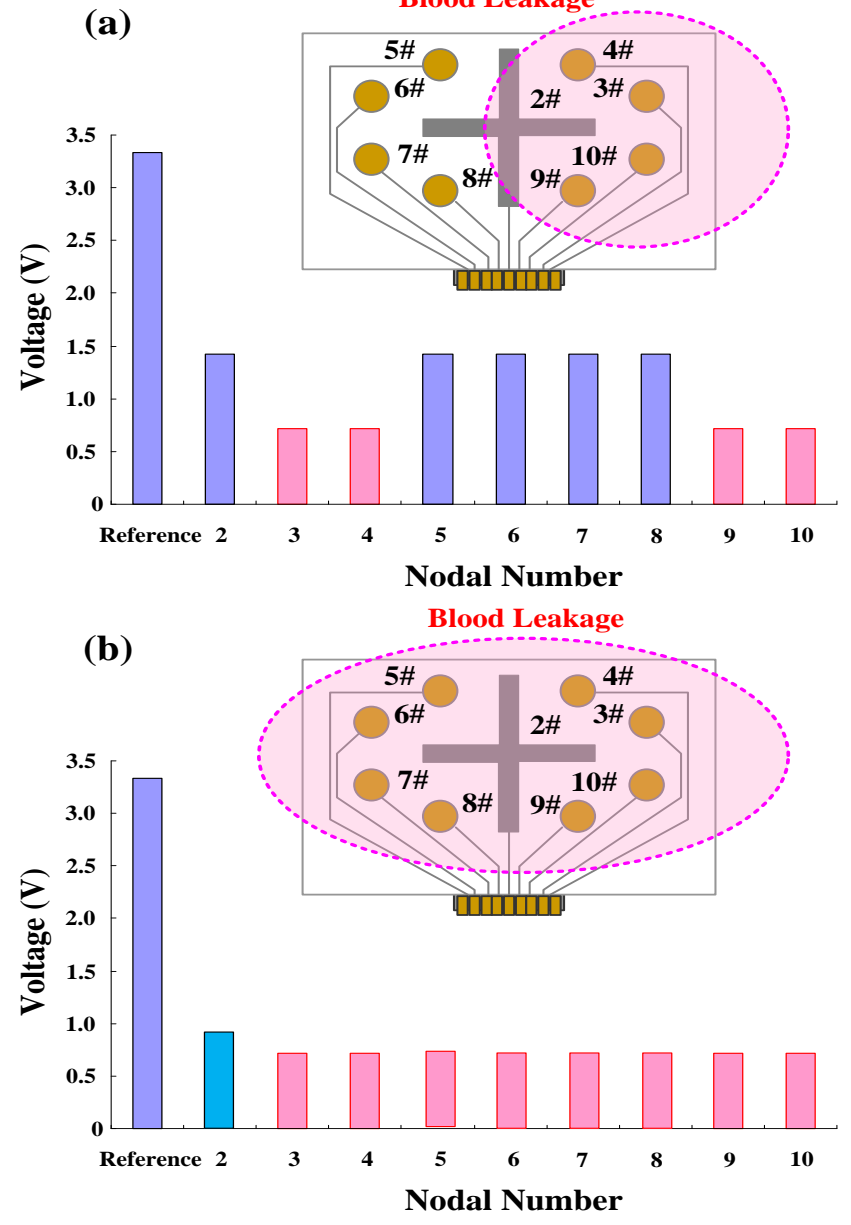

Figure 6. Voltage distributions. (a) Nodal voltage distributions for case study, (b) Nodal voltage distributions for serious case

For normal condition and eight blood leakage events, nodal voltages, $V_{2}$ to $V_{10}$, can be calculated using equations (6) to (11), and the nodal voltage distributions are illustrated in Figure 5(a). Therefore, local or global information can be characterized by the binary sensing/communication matrix using equations (12) and (13), while state signals are further used to drive an alarm unit. The nodal voltages versus blood leakage events are depicted in Figure 5(b). Nodal voltage, $V_{2}$, is regarded as a reference voltage, while the voltage levels were from $3.33 \mathrm{~V}$ to $0.91 \mathrm{~V}$. That is, the binary sensing matrix can be determined.

Suppose a blood leakage event as shown in Figure 6; blood leakage covered the nodes, 3\#, 4\#, 9\#, and 10\#, and caused the short circuit. Then, the flexible sensor detected the connecting states and transited the control signals to the

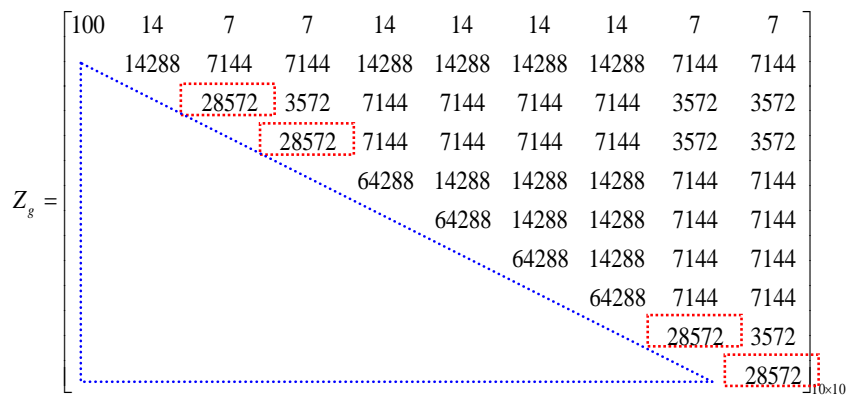

Table 1. The specification of the proposed integrating sensor

\begin{tabular}{l||l}
\hline \multicolumn{1}{l||}{ Constant Voltage Source } & $\mathrm{V}_{\mathrm{cd}}=+3.3 \mathrm{~V}$ \\
\hline \hline \multicolumn{1}{l|}{ Virtual Injection Current } & $1 \mathrm{~mA}$ \\
\hline \hline Connector & $\mathrm{MXP}$ (port) or MSP (port) \\
\hline Pull-up or Pull-down Resister & $40 \mathrm{k} \Omega$ \\
\hline \multicolumn{1}{l|}{ Wireless Communication } & $2.4 \mathrm{GHz}, \mathrm{WiFi}$, WLAN, IEEE 802.11 \\
& Standards \\
\hline Virtual Circuit Element & $R_{1}=100 \Omega, R_{\text {ref } 1}=100 \mathrm{k} \Omega, R_{\text {ref } 2}=50 \mathrm{~K} \Omega$, \\
& $L=5 \mathrm{mH}, R_{\text {ref } 4}=99 \times R_{\text {ref } 1, R_{\text {ref } 3}=1 \mathrm{~K} \Omega, R_{l}}$ \\
& $=R_{g}=50 \mathrm{~K} \Omega, l=1,2,3, \ldots, 8$ \\
\hline Voltage Level for Identifying & Virtual Voltage: $+3.33 \mathrm{~V}$ \\
Normal Condition & \\
\hline Voltage Level for Identifying & The Range of Virtual Voltage: \\
Blood Leakage / Blood Loss & $+2.50 \mathrm{~V} \sim 0.45 \mathrm{~V}$ \\
\hline Power Flow & $0.1248 \mathrm{~mW} \sim 0.0303 \mathrm{~mW}$ \\
\hline
\end{tabular}

virtual DC grid, as shown below:

$$
\left[C_{1}, C_{2}, C_{3}, C_{4}, C_{5}, C_{6}, C_{7}, C_{8}\right]=[1,1,0,0,0,0,1,1]
$$

The self-organizing algorithm acted to construct the impedance matrix, $Z_{g}$, at the top of the next page. The nodal voltages were calculated using equation (10), as shown in Figures 5 and 6(a). Their grasp drops can be identified using equations (12) and (13), as follows:

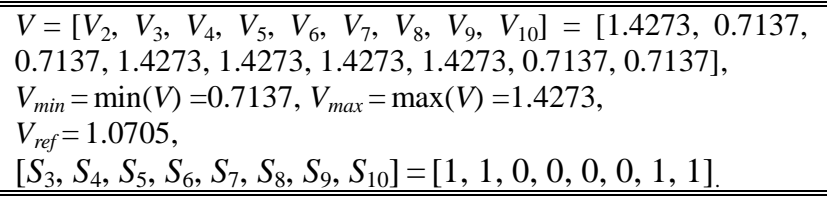

State variables indicate "signal 1" to identify the blood leakage event and to drive an alarm unit. In addition, power flow was calculated using equation (14) and its value increased from $0.0000 \mathrm{~mW}$ to $0.1019 \mathrm{~mW}$ at the grounded branches. Figure 6(b) shows the nodal voltage distributions for a serious case. Overall nodal voltages dropped and power flow also increased from $0.0000 \mathrm{~mW}$ to $0.0303 \mathrm{~mW}$. These findings confirmed that the proposed model could detect blood leakage or serious blood loss during dialysis treatment.

\section{Conclusion}

Medical electrical equipments need to be validated for safety and effectiveness before their commercialization. The standard of IEC 60601 series [20] covers the design methodology, verification, and risk assessment. Considering the biocompatibility and electrical safety, the design of the flexible sensor reduced a large amount of electrical circuits used to produce the analytical circuit and the alarm unit. 
The flexible sensor was made using a screen printing technique with elastic and metallic materials. The analytical and alarm units were virtualized and implemented in an embedded system. A self-organizing algorithm using a large matrix method was employed to construct the virtual DC grid. Its variable structure can construct a virtual DC grid for handling various conditions. Table 1 shows the specification of the proposed integrating sensor. The feasibility of the proposed prototype has been verified and this simple technique can be easily implemented on a tablet PC or a mobile appliance via wireless connection, cloud computing, and cloud storage for clinical applications.

\section{.Acknowledgement}

This work is supported in part by the Ministry of Science and Technology, Taiwan, under contract number: MOST 104-2221-E-244-003, duration: August 12015 October 31 2016, and is also supported in part by the research grant of Kaohsiung Veterans General Hospital, under contract number: VGHKS 105-070, duration: January $12016 \sim$ December 31, 2016.

\section{Reference}

[1] Jan H. M. Tordolr, "Dialysis: early pre-emptive intervention might reduce AVF access loss, "Nature Reviews, Nephrology, vol. 10, January 2014, pp. 9-10.

[2] Hans-Dietrich Polaschegg, "Venous needle dislodgement: the pitfalls of venous pressure measurement and possible alternatives, a review, "Journal of Renal Care, vol. 36, no. 1, 2010, pp. 41-48.

[3] B. Axley, J. Speranza-Reid, and H. Williams, "Venous needle dislodgement in patients on hemodialysis, "Nephrology Nursing Journal, vol. 39, no. 6, November-December 2012, pp. 435-445.

[4] American Nephrology Nurses' Association (ANNA), 2012, https://www.annanurse.org/resources/venous-needle-dislodge ment.

[5] S. Sandroni, "Venous needle dislodgement during hemodialysis: an unresolved risk of catastrophic hemorrhage," Briefing paper presented for discussion for the EDTNA/ ERCA Journal Club, 2005.

[6] R. Mactier and D. Worth, "Minimizing the risks of needle dislodgement during haemodialysis, "Artery, vol. 41, no. 3, 2007, pp. 9-13.

[7] L. C. Dinwiddie, "Vascular access for hemodialysis, "In C.S. Counts, Core curriculum for Nephrology Nursing, 2008, pp. 756-758.

[8] J. Hurst, "It can happen without warning: venous needle dislodgement, "Renal Business Today, vol. 4, no. 9, 2009, pp. 18-22.

[9] Ramesh Wariar, Thomas Hartranft, Norm Cameron, Angel Lasso, Hector Caro, Patente (US 20020198483 A1): Needle dislodgement detection, https://www.goole.ch/patents/US20 020198483.

[10] Bradlley Jon Moll, Rodney L. Moll, and Anne E. Moll Family Trust, Patente (US 20050038325 A1): Method and device for monitoring loss of body fluid and dislodgment of medical instrument from body, https://www.goole.ch/ patents/US200 $\underline{50038325}$.

[11] Marco Donati, Nicola Vitiello, Stefano Macro Maria De Rossi, Tommaso Lenzi, Simona Crea, Alessandro Persichetti, Francesco Giovacchini, Bram Koopman, Janez Podobnik, Marko Munih, and Maria Chiara Carrozza, "A flexible sensor technology for the distributed measurement of interaction pressure, "Sensors, vol. 13, 2013, pp. 1021-1045.

[12] Rita Salvado, Caroline Loss, Ricardo Goncalves, and Pedro Pinho, "Textile materials for the design of waerable antennas: a survey, "Sensors, vol. 12, 2012, pp. 15841-15857.

[13] Ruhma Tahir, Hasan Tahir, and Klaus McDonald-Maier, " Securing health sensing using integrated circuit metric," Sensors, vol.15, 2015, pp. 26621-26642.

[14] Ali Maknouninejad, Zhihua Qu, Frank L. Lewis, and Ali Davoudi, "Optimal, nonlinear, and distributed designs of droop conrols for DC microgrids, "IEEE Transactions on Smart Grid, vol. 5, no. 5, September 1014, pp. 2508- 2516.

[15] A. Maknouninejad, W. Lin, H. G. Harno, Z. Qu, and M. A. Simaan, "Cooperative control for self-organizing microgrids and game strategies for optimal dispatch of distributed renewable generations ,"Energy System, vol. 3, no. 1, 2012, pp. 23-60.

[16] Institute of Electrical and Electronics Engineers. IEEE Std. 802.11-2007, Wireless LAN Medium Access Control (MAC) and Physical Layer (PHY) Specifications, 12 June 2007.

[17] H. E. Brown, Solution of large network by matrix methods, Wiley, New York, 1975.

[18] Yan Ma and George G. Karady, "Investigating grounding grid integrity based on the current injection method, "2009 North American Power Symposium, October 4-6, 2009, pp. 1-5.

[19] Debora Rosana Ribeiro Penido, Leandro Ramos de Araujo, Sandoval Carneiro, Jose Luiz Rezende Pereira, and Paulo Augusto Nepomuceno Garcia, "Three-phase power flow based on four-conductor current injection method for unbalanced distribution networks, "IEEE Transactions on Power system, vol. 23, no. 2, May 2008, pp. 494-503.

[20] IEC 60601-1:2005, International Standard, Medical electrical equipment - Part 1: General requirements for basic safety and essential performance. 